\title{
System 3D Jones-matrix polarimetry of polycrystalline films of biological fluids
}

\author{
A. G. Ushenko, V. G. Zhytaryuk, M. I. Sidor, O. Ya. \\ Wanchulyak, A. V. Motrich, et al.
}

A. G. Ushenko, V. G. Zhytaryuk, M. I. Sidor, O. Ya. Wanchulyak, A. V. Motrich, I. V. Soltys, O. V. Pavliukovich, N. Pavliukovich, "System 3D Jones-matrix polarimetry of polycrystalline films of biological fluids," Proc. SPIE 10726, Nanoimaging and Nanospectroscopy VI, 1072613 (5 September 2018); doi: $10.1117 / 12.2320533$

Event: SPIE Nanoscience + Engineering, 2018, San Diego, California, United States 


\title{
System 3D Jones-matrix polarimetry of polycrystalline films of biological fluids
}

\author{
A.G. Ushenko ${ }^{1 *}$, V.G. Zhytaryuk ${ }^{1}$, M.I.Sidor ${ }^{1}$,O. Ya. Wanchulyak ${ }^{2}$, A.V. Motrich ${ }^{1}$, I.V. Soltys ${ }^{1}$, \\ O.V.Pavliukovich ${ }^{2}$, N.Pavliukovich ${ }^{2}$ \\ ${ }^{1}$ Chernivtsi National University, 2 Kotsiubynskyi Str., Chernivtsi, Ukraine, 58012 \\ ${ }^{2}$ Bukovinian State Medical University, 3 Theatral Sq., Chernivtsi, Ukraine, 58000
}

o.ushenko@chnu.edu.ua

\begin{abstract}
A model of multilayer polycrystalline structure of films of biological fluids of human organs has been developed.

Each layer is associated with a partial Jones-matrix operator of phase and amplitude anisotropy. A new principle for detecting polarization-inhomogeneous object fields using a coherent laser wave is proposed. Algorithms for digital holographic reconstruction of field distributions of complex amplitudes in the plane of a polycrystalline film of a biological fluid are found. A new optical technique is proposed: direct measurement of 3D distributions of elements of the Jones matrix. Maps of layer-by-layer distributions of elements of the Jones matrix of polycrystalline urine films are studied. Sensitivity, specificity and balanced accuracy of the 3D Jones-matrix tomography method of the polycrystalline structure of urine films of healthy donors and patients with albuminuria were determined. Within the framework of the statistical analysis of stratified maps of elements of the Jones matrix of polycrystalline urine films, objective criteria for the early diagnosis of the onset and course of albuminuria were found.
\end{abstract}

Keywords: Jones matrix, optical anisotropy, biological fluids, diagnostics.

\section{Jones-matrix approach in analysis of optical anisotropy of polycrystalline films of human biological liquids}

\subsection{Introduction}

The structure of biological layers ${ }^{1-10}$ can be considered as structurally inhomogeneous one ${ }^{11-20}$. In order to describe the transformation of polarized light by such complex media it is necessary to involve the most general approaches based on Mueller- or Jones-matrix formalism ${ }^{21-31}$.

Our work is devoted to the study of the possibilities of the method of polarization correlametry - determination of layered distributions modulus and phase of the Jones matrix elements within the statistical ${ }^{15,17,32-38}$ and correlation ${ }^{8,12,16,39-42}$ approaches, which characterize the optical anisotropic structures of the polycrystalline films of biological liquids почек здоровых и больных альбуминурией пациентов The purpose of the research is to determine the objective criteria that provide reliable differential Jones-matrix diagnostics of such organ pathology.

\subsection{Brief theoretical background}

In the approximation of weak phase fluctuations, the optical properties of polycrystalline films of biological fluids with a linear $(\vartheta)$ and circular $(\gamma)$ birefringence describes a Jones matrix operator of the following form ${ }^{2-5}$

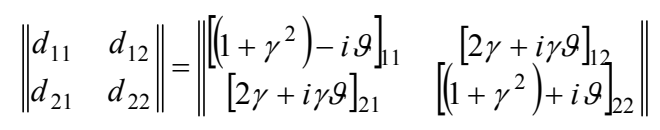

From the Jones matrix (1) we obtain analytic expressions for the modulus $\left|j_{i k}\right|$ and the phase $\operatorname{Arg}\left(j_{i k}\right)$ of complex matrix elements

Nanoimaging and Nanospectroscopy VI, edited by Prabhat Verma, Alexander Egner, Proc. of SPIE

Vol. 10726, 1072613 - (C) 2018 SPIE · CCC code: 0277-786X/18/\$18 · doi: 10.1117/12.2320533 


$$
\begin{gathered}
\left|d_{11}\right|=\left|d_{22}\right| \equiv\left|d_{11 ; 22}\right|=\left(\left(1+\gamma^{2}\right)^{2}+\vartheta^{2}\right)^{0,5} \\
\operatorname{Arg}\left(d_{11}\right)=-\operatorname{Arg}\left(d_{22}\right) \equiv \operatorname{Arg}\left(d_{11 ; 22}\right)= \pm \operatorname{arctg}\left(\frac{\vartheta}{1+\gamma^{2}}\right) \\
\left|d_{12}\right|=\left|d_{21}\right| \equiv\left|d_{12 ; 21}\right|=\gamma\left(4+\vartheta^{2}\right)^{0,5} ; \\
\operatorname{Arg}\left(j_{12}\right)=-\operatorname{Arg}\left(j_{21}\right) \equiv \operatorname{Arg}\left(j_{12 ; 21}\right)= \pm \operatorname{arctg}\left(\frac{\delta}{2}\right)
\end{gathered}
$$

Obtaining relations (2) - (5) represent the theoretical basis of Jones-matrix tomography - polarization restoration of the distributions of phase anisotropy parameters of the polycrystalline structure of biological layers.

\subsection{Method of measuring the elements of the Jones-matrix and the optical scheme of polarization correlometry}

In fig. 1 shows an optical scheme of polarization correlometry ${ }^{38-42}$ of laser microscopic images of biological layers.



Fig. 1. Optical scheme of polarization interferometry of microscopic images of optically anisotropic biological layers. Explanation in the text

The parallel $\left(\varnothing=2 \times 10^{3} \mu \mathrm{m}\right)$ beam He-Ne $(\lambda=0,6328 \mu \mathrm{m})$ of laser 1 , formed with the help of a collimator 2 , is divided into "irradiating" and "supporting" with $50 \%$ of the light distributor 3 . The "illuminating" using the rotating mirror 4 is routed through a polarization filter 6 to 8 in the direction of the sample of the biological layer 9. The polarizationheterogeneous image of the object 9 by the lens 10 is projected into the plane of the digital camera 14. The "reference" beam with the mirror 5 is rotated through the mirror through the polarization filter 11 - 13 in the plane of the polarization-heterogeneous image of the object 9. As a result, an interference pattern is formed, the coordinate distribution of the intensity of which the digital camera registers. 14. Formation of states of polarization " prominyuyuchoho "and" reference "beam by means of polarization filters $6-8$ and 11 - 13. Each of these filters comprises:

- "input" polarizers 6 and 11, which form flat-polarized beam with azimuth $\alpha_{0}=0^{0}$;

- Quaternary plates 7 and 12, which form right circularly polarized beams $\beta_{0}=+45^{0}$;

- "output" polarizers 8 and 13, which, due to the rotation of the transmission plane, are formed without a change in intensity, flat-polarized with an arbitrary azimuth $0^{0} \leq \alpha_{0} \leq 180^{\circ}$ of the beam. 


\subsection{Polarization -correlation Jones-matrix mapping}

The method of polarization-correlation determination of the set of matrix elements consists in the following set of actions:

- Simultaneous formation of "laser" and "reference" parallel laser beams of two states of polarization $\left(\left(0^{0}-0^{0}\right) ; \quad\left(90^{0}-90^{0}\right)\right.$.

- Registration of each partial interference pattern through a polarizer-analyzer with orientation of the plane of passage at angles $\Theta=0^{\circ} ; \quad \Theta=90^{\circ}$.

- Recovery for each partial interference distribution using the integrated diffraction transformation of the coordinate distributions of the complex amplitudes $\left\{U_{x}(x, y) ; u_{y}(x, y)\right\}$ of the object field in the plane of the biological layer ${ }^{38}$.

An analytical algorithm for computing the values of elements of the Jones matrix describes the following relationships:

$$
U^{*}\left(0^{0}\right)=\{D\} U\left(0^{0}\right)=\left(\begin{array}{l}
U_{x}^{*} \\
U_{y}^{*}
\end{array}\right)=\left\|\begin{array}{ll}
d_{11} & d_{12} \\
d_{21} & d_{22}
\end{array}\right\|\left(\begin{array}{l}
1 \\
0
\end{array}\right) \Rightarrow\left(\begin{array}{l}
d_{11} \\
d_{21}
\end{array}\right) ;
$$

A beam of light with Jones vector $U^{*}\left(0^{0}\right)$ passes through a polarizer-analyzer with the orientation of the transmission plane $\Omega=0^{0} ; 90^{\circ}$

$$
\begin{gathered}
U_{x}^{*}\left(0^{0}\right)=\left\|\begin{array}{ll}
1 & 0 \\
0 & 0
\end{array}\right\|\left(\begin{array}{l}
d_{11} \\
d_{12}
\end{array}\right) \Rightarrow d_{11} ; \\
U_{y}^{*}\left(0^{0}\right)=\left\|\begin{array}{ll}
0 & 0 \\
0 & 1
\end{array}\right\|\left(\begin{array}{l}
d_{11} \\
d_{12}
\end{array}\right) \Rightarrow j_{21} ; \\
U^{*}\left(90^{0}\right)=\{D\} U\left(90^{0}\right)=\left(\begin{array}{l}
U_{x}^{*} \\
U_{y}^{*}
\end{array}\right)=\left\|\begin{array}{ll}
d_{11} & d_{12} \\
d_{21} & d_{22}
\end{array}\right\|\left(\begin{array}{l}
0 \\
1
\end{array}\right) \Rightarrow\left(\begin{array}{l}
d_{21} \\
d_{22}
\end{array}\right) ;
\end{gathered}
$$

Like the case (6), a beam of light with a Jones vector $U^{*}\left(90^{\circ}\right)$ passes through a polarizer-analyzer with the orientation of the transmission plane $\Omega=0^{0} ; 90^{0}$

$$
\begin{aligned}
& u_{x}^{*}\left(90^{0}\right)=\left\|\begin{array}{ll}
1 & 0 \\
0 & 0
\end{array}\right\|\left(\begin{array}{l}
d_{21} \\
d_{22}
\end{array}\right) \Rightarrow d_{21} ; \\
& U_{y}^{*}\left(0^{0}\right)=\left\|\begin{array}{ll}
0 & 0 \\
0 & 1
\end{array}\right\|\left(\begin{array}{l}
d_{21} \\
d_{22}
\end{array}\right) \Rightarrow d_{22} .
\end{aligned}
$$

Consequently, the collection of complex elements of the Jones matrix obtained by direct polarization-correlation measurement can be used as a basis for obtaining algorithms for determining the parameters of phase anisotropy. To this end, we use the explicit form of the theoretical Jones matrix of an optically anisotropic layer with a linear $(\vartheta)$ and circular $(\gamma)$ birefringence refraction. You can show that these parameters are determined by the following relationships

$$
\vartheta=\frac{\arccos \left[\operatorname{Re}\left(d_{11}+d_{22}\right)\right]}{\left[\left(\operatorname{Re} d_{21}\right)^{2}+\left(\operatorname{Im} d_{22}\right)^{2}\right]^{0,5}} \operatorname{Im} d_{22} ;
$$




$$
\gamma=\frac{\arccos \left[\operatorname{Re}\left(d_{11}+d_{22}\right)\right]}{\left[\left(\operatorname{Re} d_{21}\right)^{2}+\left(\operatorname{Im} d_{22}\right)^{2}\right]^{0,5}} \frac{\left(\operatorname{Im} d_{22}\right)^{2}}{\operatorname{Re} d_{21}} .
$$

For optically thin layers, the algorithms of polarization-phase reconstruction in conditions of weak phase modulation $\left(\vartheta, \gamma \prec 6^{0} \rightarrow \sin (\vartheta, \gamma) \approx \vartheta, \gamma ; \cos (\vartheta, \gamma) \approx 1\right)$ acquire a simpler form

$$
\begin{gathered}
\vartheta=\operatorname{Im}\left(d_{22}-d_{11}\right) ; \\
\gamma=\operatorname{Re}\left(d_{12}\right) .
\end{gathered}
$$

\subsection{Analysis and discussion of experimental data}

\subsubsection{Polycrystalline films of biological fluids}

On the series of fig.. 2 - fig. 5 shows the results of polarization-correlation studies of the volume-layer variation of the linear $(\vartheta)$ and circular $(\gamma)$ birefringence of polycrystalline films of biological fluids characterized by distribution of magnitude in the phase cross sections $(\Delta \varphi=\Delta(\vartheta+\gamma))$ of the module $\left[d_{11}(\varphi)\right]\left[(m \times n)\right.$ and the phase $\left[\operatorname{Arg}\left(d_{11}(\varphi)\right)\right](m \times n)$ of complex elements of the Jones matrix of non-depolarizing layers of bile (fig. 2 - fig. 3) the liquor (fig. 4 - fig. 5).

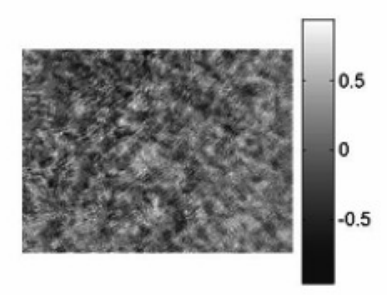

(1)

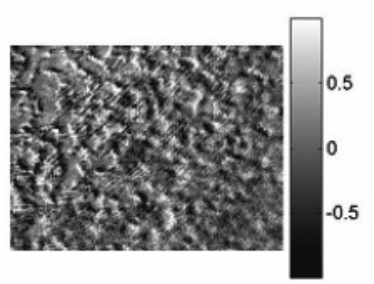

(3)



(2)



(4)

Fig. 2. By layered planar distributions of the values of the module $\left|d_{11}\right|$ of elements of the matrix of Jones polycrystalline bile films 




(1)

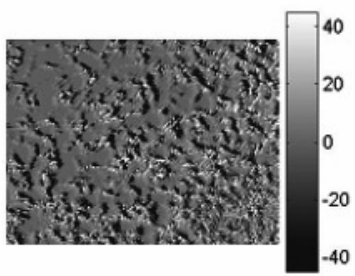

(3)

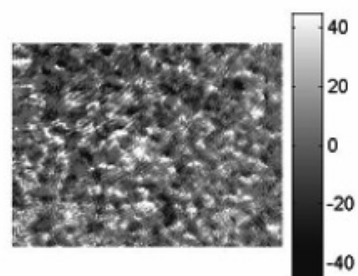

(2)

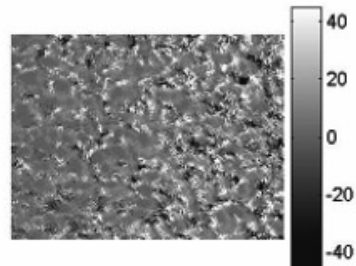

(4)

Fig. 3. By layered planar distributions of the phase $\operatorname{Arg}\left(d_{11}\right)$ values of elements of the Jones matrix of polycrystalline bile films.

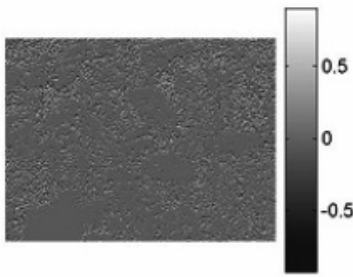

(1)

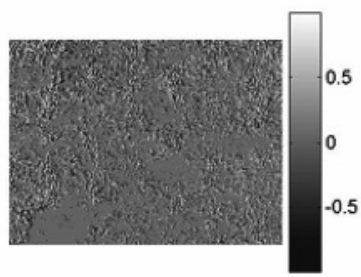

(3)



(2)

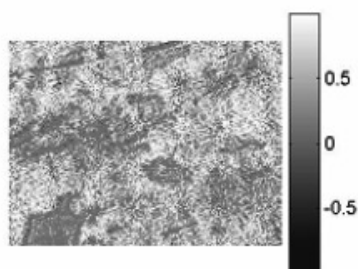

(4)

Fig. 4. By layered planar distributions of values of the module $\left|d_{11}\right|$ of elements of the matrix of Jones polycrystalline cerebral film. 




(1)

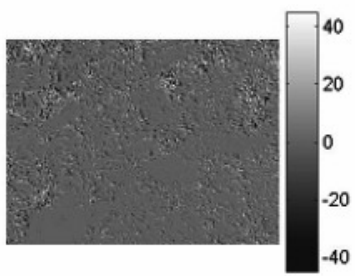

(3)

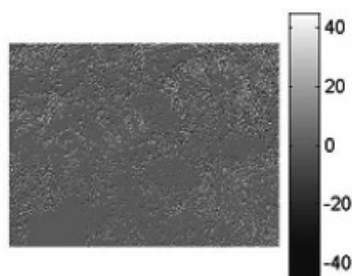

(2)



(4)

Fig. 5. By layered planar distributions of the values of the phase $\operatorname{Arg}\left(d_{11}\right)$ of elements of the matrix of the Jones polycrystalline cerebral film.

The analysis of the data obtained revealed significant volumetric homogeneity of the coordinate distribution of the magnitude $\left[d_{11}(\varphi)\right](m \times n)$ and $\left[\operatorname{Arg}\left(d_{11}(\varphi)\right)\right](m \times n)$ characterizing the polycrystalline structure of the bile films (Figures 3 and 4) and the liquor (Figs 4 and 5). On this fact, there are significant changes in the coordinate structure of the data of components of the complex elements of the Jones matrix, which are obtained for different phase cross sections $\varphi=0.5 \mathrm{rad} ; 0.75 \mathrm{rad} ; 1 . \mathrm{rad} ; 1.25 \mathrm{rad}$.

The physics of the detection of patterns of random variation and coordinate heterogeneity of distributions $\left[d_{11}(\varphi)\right](m \times n)$ and $\left[\operatorname{Arg}\left(d_{11}(\varphi)\right)\right](m \times n)$, it is possible to link with the fact that the volumetric polycrystalline structure of optically thin films of biological fluids is formed in a layered, step by step manner. Due to this, fluctuations of the phase arise in the volume of polycrystalline films, which consist of optically anisotropic compounds and networks of biochemical crystals.

\section{Clinical application of the Jones-matrix cartography method}

\subsection{Jones-matrix differential diagnosis of weak changes in optical anisotropy of polycrystalline films of human organs}

The results of the method of 3D - Jones matrix mapping of distributions of values of the module $\left|d_{11}\right|$ and $A r g\left(d_{11}\right)$ the phase of polycrystalline films of biological liquids in the differentiation of weak changes in phase anisotropy caused by pathology of various degrees of gravity are presented.

Method of differentiation of samples of polycrystalline liquids of human organs (knee joint, kidneys) with different pathology (synovitis, albuminuria) consisted of:

- Determination of the set of layered phase cross sections of the 3D distributions of the module $\| d_{11 ; 12}(\varphi) \mid(m \times n)$ and the phases $\left[\operatorname{Arg}\left(d_{11 ; 12}(\varphi)\right)(m \times n)\right.$ of the complex elements of the Jones matrix;

- computing a set of statistical $P_{3 ; 4}(\varphi)$ moments for each section of the module $\left\|d_{11 ; 12}(\varphi)\right\|(m \times n)$ and phase $\left.\operatorname{Arg}\left(d_{11 ; 12}(\varphi)\right)\right](m \times n)$ distributions;

- Identifying the maximum differences between statistical $\Delta P_{3 ; 4}(\varphi)=\max$ moments; 
- calculations within the defined partial layer of mean values $\bar{P}_{3 ; 4}(\varphi)$ and their errors $\pm \sigma$ for each group of samples of polycrystalline films of biological liquids of organs of human with varying degrees of severity of pathology;

- Finding a balanced diagnostic test $A c, \%$ based on a Jones-matrix mapping using a reference laser wave.

The results of experimental studies of the 3D diagnostic efficiency of the Jones-matrix mapping of the modulus $\| d_{11 ; 12}(\varphi) \mid(m \times n)$ and phase $\left[\operatorname{Arg}\left(d_{11 ; 12}(\varphi)\right)(m \times n)\right.$ distributions characterizing the phase anisotropy of synovial fluid and urine polycrystalline films are given in table $1\left(\left[d_{11}(\varphi)\right](m \times n)\right.$ i $\left.\left[\operatorname{Arg}\left(d_{11}(\varphi)\right)\right](m \times n)\right)$ and table 2 $\left(\llbracket d_{12}(\varphi)\right](m \times n)$ and $\left.\left[\operatorname{Arg}\left(d_{12}(\varphi)\right)\right)(m \times n)\right)$.

Table 1. Balanced accuracy of 3D method - Jones matrix mapping of distribution of values of module $\left|d_{11}\right|$ and phase $\operatorname{Arg}\left(d_{11}\right)$ of polycrystalline films of biological fluids

\begin{tabular}{|c|c|c|c|c|}
\hline Parameters & \multicolumn{2}{|c|}{$\left|d_{11}\right|$} & \multicolumn{2}{|c|}{$\operatorname{Arg}\left(d_{11}\right)$} \\
\hline$P_{i}, d_{11}, \operatorname{Arg}\left(d_{11}\right)$ & $\begin{array}{c}\text { Synovial fluid, } \\
A c, \%\end{array}$ & $\begin{array}{c}\text { Urine, } \\
A c, \%\end{array}$ & $\begin{array}{c}\text { Synovial fluid, } \\
A c, \%\end{array}$ & $\begin{array}{c}\text { Urine, } \\
A c, \%\end{array}$ \\
\hline$P_{1}$ & 73 & 67 & 96 & 81 \\
\hline$P_{2}$ & 76 & 79 & 79 & 72 \\
\hline$P_{3}$ & 84 & 73 & 85 & 83 \\
\hline$P_{4}$ & 94 & 82 & 92 & 80 \\
\hline
\end{tabular}

Table 2. Balanced accuracy of 3D method - Jones matrix mapping of distribution of values of module $\left|d_{12}\right|$ and phase $A r g\left(d_{12}\right)$ of polycrystalline films of biological fluids

\begin{tabular}{|c|c|c|c|c|}
\hline Parameters & $\left|d_{12}\right|$ & \multicolumn{2}{|c|}{$\operatorname{Arg}\left(d_{12}\right)$} \\
\hline$P_{i}, d_{12}, \operatorname{Arg}\left(d_{12}\right)$ & Synovial fluid, $A c, \%$ & $\begin{array}{l}\text { Urine, } \\
A c, \%\end{array}$ & Synovial fluid, $A c, \%$ & $\begin{array}{c}\text { Urine, } \\
A c, \%\end{array}$ \\
\hline$P_{1}$ & 78 & 63 & 96 & 82 \\
\hline$P_{2}$ & 81 & 72 & 91 & 80 \\
\hline$P_{3}$ & 85 & 74 & 92 & 81 \\
\hline$P_{4}$ & 91 & 77 & 95 & 83 \\
\hline
\end{tabular}

From the obtained data it is seen that the use of the 3D Jones matrix tomography method provides an opportunity for efficient differentiation (Table 1 and Table 2, highlighted by gray) of pathological changes in the polycrystalline structure of biological fluid films:

- Synovial fluid of the joint of a person with excellent balanced accuracy ( $A c \succ 95 \%$ );

- urine of healthy and patients with albuminuria of patients with good balanced accuracy ( $A c \sim 85 \%$ )

\section{Conclusions}

1. The method of azimuthally invariant 3D Jones matrix mapping of the distribution of phase anisotropy parameters of polycrystalline films of biological fluids is proposed and grounded.

2. The coordinates of the magnitude of the module and the phase of the complex of elements of the Jones matrix polycrystalline films of bile and liquor are obtained in the volume of biological samples. 
3. The "phase" dependences of the magnitude of the statistical moments of the 1st-4th orders, which characterize the distributions of the values of the module and the phases of the elements of the Jones matrix of the synovial fluid of the knee joint and urine, are determined in the presence of knee joint and kidney pathology.

4. The optimal conditions for differentiation of polycrystalline structures of biological tissues - the range of phase cross sections $(0,3 \mathrm{rad} \leq \varphi \leq 0,9 \mathrm{rad})$ and the most sensitive parameters - are the statistical moments of the 3rd and 4th orders, which characterize the distributions of the module and phases of complex elements of the Jones matrix of different types of optically anisotropic structures of films of biological fluids human organs.

\section{References}

[1] De Martino, Ed., “A polarization-based optical techniques applied to biology and medicine,” in Proc. European Workshop, Ecole Polytechnique, Massy, France (2009).

[2] N. Ghosh and I. A. Vitkin, "Tissue polarimetry: concepts, challenges, applications and outlook," J. Biomed. Opt. 16, 110801 (2011).

[3] S. L. Jacques, "Polarized light imaging of biological tissues" in Handbook of Biomedical Optics, D. Boas, C. Pitris, and N. Ramanujam, Eds., pp. 649-669, CRC Press, Boca Raton, London, New York (2011).

[4] N. Ghosh, M. F. G. Wood, and I. A. Vitkin, "Polarized light assessment of complex turbid media such as biological tissues via Mueller matrix decomposition," in Handbook of Photonics for Biomedical Science, V. V. Tuchin, Ed., pp. 253-282, CRC Press, Taylor \& Francis Group, London (2010).

[5] D. Layden, N. Ghosh, and A. Vitkin, "Quantitative polarimetry for tissue characterization and diagnosis," in Advanced Biophotonics: Tissue Optical Sectioning, R. K. Wang and V. V. Tuchin, Eds., pp. 73-108, CRC Press, Taylor \& Francis Group, Boca Raton, London, New York (2013).

[6] A. Vitkin, N. Ghosh, and A. de Martino, "Tissue polarimetry" in Photonics: Scientific Foundations, Technology and Applications, D. L. Andrews, Ed., Vol. IV, pp. 239-321, John Wiley \& Sons, Inc., Hoboken, New Jersey (2015).

[7] M. I. Mishchenko, L. D. Travis, and A. A. Lacis, Scattering, Absorption, and Emission of Light by Small Particles, Cambridge University Press, Cambridge (2002).

[8] M. K. Swami, H. S. Patel, and P. K. Gupta, "Conversion of $3 \times 3$ Mueller matrix to $4 \times 4$ Mueller matrix for non-depolarizing samples," Opt. Commun. 286(1), 18-22 (2013).

[9] V. F. Izotova et al., "Investigation of Mueller matrices of anisotropic nonhomogeneous layers in application to optical model of cornea," Appl. Opt. 36(1), 164-169 (1997).

[10] V. V. Tuchin, "Tissue optics and photonics: biological tissue structures [review]," J. Biomed. Photonics Eng. 1(1), 3-21 (2015).

[11] V. V. Tuchin, "Tissue optics and photonics: light-tissue interaction [review],” J. Biomed. Photonics Eng. 1(2), 98-134 (2015).

[12] S. Bartel and A. H. Hielscher, "Monte Carlo simulations of the diffuse backscattering Mueller matrix for highly scattering media," Appl. Opt. 39, 1580-1588 (2000).

[13] X. Wang and L. V. Wang, "Propagation of polarized light in birefringent turbid media: a Monte Carlo study," J. Biomed. Opt. 7(3), 279-290 (2002).

[14] M.-R. Antonelli et al., "Impact of model parameters on Monte Carlo simulations of backscattering Mueller matrix images of colon tissue," Biomed. Opt. Express 2(7), 1836-1851 (2011).

[15] M. R. Antonelli et al., "Mueller matrix imaging of human colon tissue for cancer diagnostics: how Monte Carlo modeling can help in the interpretation of experimental data," Opt. Express 18(10), 10200- 10208 (2010).

[16] I. A. Vitkin and R. C. N. Studinski, "Polarization preservation in diffusive scattering from in vivo turbid biological media: effects of tissue optical absorption in the exact backscattering direction," Opt. Commun. 190, 37-43 (2001).

[17] Y. P. Sinichkin et al., "Reflectance and fluorescence spectroscopy of human skin in vivo," in Handbook of Optical Biomedical Diagnostics, 2nd ed., V. V. Tuchin, Ed., pp. 95-185, SPIE Press, Bellingham, Washington (2016).

[18] L. T. Perelman and V. Backman, "Light scattering spectroscopy of epithelial tissues: principles and applications," in Handbook of Optical Biomedical Diagnostics, 2nd ed., V. V. Tuchin, Ed., pp. 33-93, SPIE Press, Bellingham, Washington (2016).

[19] S. Manhas et al., "Mueller matrix approach for determination of optical rotation in chiral turbid media in backscattering geometry,” Opt. Express 14(1), 190-202 (2006).

[20] Y. Deng et al., "Characterization of backscattering Mueller matrix patterns of highly scattering media with triple scattering assumption," Opt. Express 15(15), 9672-9680 (2007).

[21] Alexander G. Ushenko and Vasilii P. Pishak, "Laser Polarimetry of Biological Tissue: Principles and Applications", in Handbook of Coherent-Domain Optical Methods: Biomedical Diagnostics, Environmental and Material Science, vol. I, Valery V. Tuchin, Ed. Boston: Kluwer Academic Publishers, 2004, pp. 93-138.

[22] O. V. Angelsky, A. G. Ushenko, Yu. A. Ushenko, V. P. Pishak, A. P. Peresunko, "Statistical, Correlation and Topological Approaches in Diagnostics of the Structure and Physiological State of Birefringent Biological Tissues" in Handbook of Photonics for Biomedical Science, pp. 283-322 ed. by Valery V. Tuchin, CRC PressTaylor\&Francis group: Boca Raton, London, New York (2010). 
[23] Y. A. Ushenko, T. M. Boychuk, V. T. Bachynsky, O. P. Mincer, "Diagnostics of Structure and Physiological State of Birefringent Biological Tissues: Statistical, Correlation and Topological Approaches" in Handbook of Coherent-Domain Optical Methods, Springer Science+Business Media, p. 107, New York (2013).

[24] S. Y. Lu and R. A. Chipman, "Interpretation of Mueller matrices based on polar decomposition," J. Opt. Soc. Am. A 13(5), 1106- 1113 (1996).

[25] Y. Guo et al., "A study on forward scattering Mueller matrix decomposition in anisotropic medium," Opt. Express 21(15), 18361-18370 (2013).

[26] B. Deboo, J. Sasian, and R. A. Chipman, "Degree of polarization surfaces and maps for analysis of depolarization," Opt. Express 12(20), 4941-4958 (2004).

[27] I. C. Buscemi and S. Guyot, "Near real-time polarimetric imaging system," J. Biomed. Opt. 18(11), 116002 (2013).

[28] S. Manhas et al., "Demonstration of full $4 \times 4$ Mueller polarimetry through an optical fiber for endoscopic applications," Opt. Express 23(3), 3047-3054 (2015).

[29] A. Pierangelo et al., "Multispectral Mueller polarimetric imaging detecting residual cancer and cancer regression after neoadjuvant treatment for colorectal carcinomas," J. Biomed. Opt. 18(4), 046014 (2013).

[30] L. V. Wang and H.-I. Wu, Biomedical Optics: Principles and Imaging, Wiley-Interscience, Hoboken, New Jersey (2007).

[31] D. Boas, C. Pitris, and N. Ramanujam, Eds., Handbook of Biomedical Optics, CRC Press, Boca Raton, London, New York (2011).

[32] T. Vo-Dinh, Ed., Biomedical Photonics Handbook, 2nd ed., CRC Press, Boca Raton (2014).

[33] V. V. Tuchin, Tissue Optics: Light Scattering Methods and Instruments for Medical Diagnostics, 3rd ed., Vol. PM 254, SPIE Press, Bellingham, Washington (2015).

[34] D. K. Kasaragod et al., "Experimental validation of an extended Jones matrix calculus model to study the 3D structural orientation of the collagen fibers in articular cartilage using polarization-sensitive optical coherence tomography," Biomed. Opt. Express 3(3), 378-387 (2012).

[35] Y. Yasuno et al., "Jones Matrix based polarization sensitive optical coherence tomography," in Optical Coherence Tomography: Technology and Applications, 2nd ed., W. Drexler and J. G. Fujimoto, Eds., pp. 1137-1162, Springer Reference, Science + Business Media, New York (2015)

[36] Lu S. Interpretation of Mueller matrices based on polar decomposition / S. Lu, R. A. Chipman // J. Opt. Soc. Am. A, Vol.13, 1106-1113, 1996.

[37] Ghosh N. Polarized light assessment of complex turbid media such as biological tissues using Mueller matrix decomposition / N. Ghosh, M. Wood, and A. Vitkin // Handbook of Photonics for Biomedical Science; Ed. by V. V. Tuchin. - London : Taylor and Francis Publishing. - 2010. - Chapter 9. - P. 253-282

[38] Ushenko V. A. Azimuthally invariant Mueller-matrix mapping of biological tissue in differential diagnosis of mechanisms protein molecules networks anisotropy / V. A. Ushenko, M. S. Gavrylyak . // Proc. SPIE 8812, Biosensing and Nanomedicine VI, 88120Y (September 11, 2013).

[39] Angelsky, O.V., Maksimyak, P.P., Ryukhtin, V.V., Hanson, S.G., "New feasibilities for characterizing rough surfaces by optical-correlation techniques," Applied Optics, 40 (31), 5693-5707 (2001).

[40] Angelsky, O.V., Maksimyak, P.P., "Optical diagnostics of random phase objects,” Applied Optics, 29 (19), $2894-2898$ (1990).

[41] Angelsky, O. V., Bekshaev, A. Ya., Maksimyak, P. P., Maksimyak, A. P., Hanson, S. G., and Kontush, S. M., "Controllable generation and manipulation of micro-bubbles in water with absorptive colloid particles by CW laser radiation," Opt. Express 25, 5232-5243 (2017).

[42] O.V. Angelsky, S.G. Hanson, P.P. Maksimyak, A.P. Maksimyak, C.Yu. Zenkova, P.V. Polyanskii, and D.I. Ivanskyi, "Influence of evanescent wave on birefringent microplates," Opt. Express 25, 2299-2311 (2017). 